\title{
No Evidence for Neural Overlap between Unconsciously Processed and Imagined Stimuli
}

\author{
(D) Nadine Dijkstra, ${ }^{1,2}$ Simon van Gaal, ${ }^{3}$ Linda Geerligs, ${ }^{1}$ Sander E. Bosch, ${ }^{1}$ and \\ Marcel A. J. van Gerven ${ }^{1}$ \\ https://doi.org/10.1523/ENEURO.0228-21.2021 \\ ${ }^{1}$ Donders Institute for Brain, Cognition and Behaviour, Radboud University, 6500 GL, Nijmegen, The Netherlands, \\ ${ }^{2}$ Wellcome Centre for Human Neuroimaging, University College London, London WC1N 3AR, United Kingdom, and \\ ${ }^{3}$ Department of Psychology, Brain \& Cognition, University of Amsterdam, 1000 GG, Amsterdam, The Netherlands
}

\begin{abstract}
Visual representations can be generated via feedforward or feedback processes. The extent to which these processes result in overlapping representations remains unclear. Previous work has shown that imagined stimuli elicit similar representations as perceived stimuli throughout the visual cortex. However, while representations during imagery are indeed only caused by feedback processing, neural processing during perception is an interplay of both feedforward and feedback processing. This means that any representational overlap could be because of overlap in feedback processes. In the current study, we aimed to investigate this issue by characterizing the overlap between feedforward- and feedback-initiated category representations during imagined stimuli, conscious perception, and unconscious processing using fMRI in humans of either sex. While all three conditions elicited stimulus representations in left lateral occipital cortex (LOC), significant similarities were observed only between imagery and conscious perception in this area. Furthermore, connectivity analyses revealed stronger connectivity between frontal areas and left LOC during conscious perception and in imagery compared with unconscious processing. Together, these findings can be explained by the idea that longrange feedback modifies visual representations, thereby reducing representational overlap between purely feedforward- and feedback-initiated stimulus representations measured by fMRI. Neural representations influenced by feedback, either stimulus driven (perception) or purely internally driven (imagery), are, however, relatively similar.
\end{abstract}

Key words: consciousness; mental imagery; perception; unconscious processing

\section{Significance Statement}

Previous research has shown substantial neural overlap between imagery and perception, suggesting overlap between bottom-up and top-down processes. However, because conscious perception also involves top-down processing, this overlap could instead reflect similarity in feedback processes. In this study, we showed that the overlap between perception and imagery disappears when stimuli are rendered unconscious via backward masking, suggesting reduced overlap between purely bottom-up and top-down generated representations.

\section{Introduction}

Visual experience relies on neural representations in visual cortex, which can be activated in two different ways: externally, by light bouncing off objects and hitting

Received May 20, 2021; accepted September 2, 2021; First published September 29, 2021.

The authors declare no competing financial interests. the retina, from which signals are sent via feedforward connections to early visual cortex (EVC) and areas further up in the visual hierarchy [e.g., lateral occipital cortex (LOC)]; or internally, via feedback signals from high-level

Author contributions: N.D., S.v.G., S.E.B., and M.A.J.v.G. designed research; N.D. and S.E.B. performed research; N.D., S.v.G., and L.G. analyzed data; N.D., S.v.G., L.G., S.E.B., and M.A.J.v.G. wrote the paper. 
brain areas, such as areas in prefrontal cortex, for example, during mental imagery and dreaming (Mechelli et al., 2004; Dentico et al., 2014; Dijkstra et al., 2017b). It remains unclear to what extent activation patterns in visual cortex caused by feedforward and feedback signals are similar.

Previous work has compared neural representations during perception and imagery, revealing convincing evidence that there is neural representational overlap between perception and imagery throughout large parts of visual cortex (O'Craven and Kanwisher, 2000; Thirion et al., 2006; Stokes et al., 2009; Reddy et al., 2010; Cichy et al., 2012; Lee et al., 2012; Albers et al., 2013; Johnson and Johnson, 2014; Dijkstra et al., 2017a; Horikawa and Kamitani, 2017). The strongest overlap between perception and imagery is typically observed in high-level visual areas (Stokes et al., 2009; Reddy et al., 2010; Lee et al., 2012), whereas the overlap in low-level areas seems to depend on the required detail of the imagery task (Kosslyn and Thompson, 2003) and the experienced imagery vividness (Lee et al., 2012; Albers et al., 2013; Dijkstra et al., 2017a,b).

However, while activation in visual cortex during mental imagery indeed only relies on feedback signals (Mechelli et al., 2004; Dijkstra et al., 2017a,b, 2020), visual activation during perception reflects an interplay between feedforward and feedback processes (Lamme and Roelfsema, 2000; Muckli, 2010; Bastos et al., 2012, 2015; Dijkstra et al., 2017a,b, 2020). To determine whether visual representations activated by feedforward and feedback signals do indeed activate similar neural populations, one needs to investigate a situation in which visual representations are caused by feedforward signals only and compare those to events that include feedback processing as well.

Backward masking has been hypothesized to disrupt feedback from high-level visual cortex to early visual cortex (Lamme et al., 2002; Roelfsema et al., 2002; Del Cul et al., 2007; Fahrenfort et al., 2007; van Gaal and Lamme, 2012). In a backward-masking paradigm, a briefly presented target stimulus is rapidly followed by a second masking stimulus. Appropriate backward masking renders the target stimulus invisible. Several studies have shown that masking leaves the feedforward sweep relatively unaffected, which can still cause activation in highlevel visual cortex (Jiang and He, 2006; Sterzer et al., 2008), while feedback processing is disrupted (Lamme et al., 2002; Fahrenfort et al., 2007; van Gaal and Lamme, 2012; Mashour et al., 2020). These and other observations have led to the idea that the feedforward sweep is

N.D. was supported by Rubicon Grant 019.192SG.003; M.A.J.v.G. and S.E. B. were supported by VIDI Grant 639.072.513; and L.G. was supported by VENI Grant 451.1.013, all from the Netherlands Organization for Scientific Research.

Correspondence should be addressed to Nadine Dijkstra at n.dijkstra@ ucl.ac.uk.

https://doi.org/10.1523/ENEURO.0228-21.2021

Copyright $@ 2021$ Dijkstra et al.

This is an open-access article distributed under the terms of the Creative Commons Attribution 4.0 International license, which permits unrestricted use, distribution and reproduction in any medium provided that the original work is properly attributed. unconscious and that recurrent processing is an important factor in achieving conscious awareness (Tononi, 2008; Lamme, 2015; Mashour et al., 2020). However, the exact relationship between feedback processing and conscious awareness is still debated (Boly et al., 2017).

In the current study, we investigated to what extent visual representations in visual cortex are modified by feedback by comparing conditions in which stimuli are consciously perceived, not consciously perceived, and imagined. We rely on the assumption that unconscious processing contains less or no feedback processing, and that therefore comparing unconscious to conscious and imagined representations will provide insight into the effects of feedback processing. However, it is important to note that this is an assumption based on previous research that will not be tested in the current study. Therefore, the exact implications of our results need to be inferred with caution. More elaborate and nuanced interpretations will be given in the Discussion. We quantified the representational overlap between the different conditions by training a classifier on one condition and testing it on another condition ("cross-decoding"; Lee et al., 2012; Albers et al., 2013; Dijkstra et al., 2018). The only difference between the conscious and unconscious condition was the stimulus onset asynchrony (SOA) between the target and the mask. To cue visual imagery in a way that does not induce an informative cue signal that can be picked up by a classifier, we used a retro-cue paradigm (Harrison and Tong, 2009; Fig. 1B).

\section{Materials and Methods}

\section{Participants}

Thirty-seven participants with normal or corrected-tonormal vision gave written informed consent and participated in the study. All participants were naive to the aim of the experiment, and most participants were familiar with similar visual perception fMRI studies. Two participants were excluded from the final analyses: one because they quit the experiment prematurely and one because they had misunderstood the task. Because of an accidental change in the refresh rate of the monitor (from $60 \mathrm{to} 75 \mathrm{~Hz}$ ), the timing was slightly different for 6 of 35 participants [presentation from 17 to $13 \mathrm{~ms}$, conscious interstimulus interval (ISI) from 66 to $80 \mathrm{~ms}]$, so that the presentation times were slightly shorter for the unconscious condition and slightly longer for the conscious condition. Because this error did not change visibility ratings [unconscious: $1.37(\mathrm{SD}=0.27)$ vs $1.35(\mathrm{SD}=0.58$; $\left.t_{(33)}=0.079, p=0.94\right)$; conscious: $2.92(\mathrm{SD}=0.37)$ vs 2.98 $\left.\left(\mathrm{SD}=0.61 ; t_{(33)}=-0.25, p=0.80\right)\right]$ or discrimination sensitivity (unconscious: $0.19(\mathrm{SD}=0.28)$ vs $0.03\left(\mathrm{SD}=0.18 ; t_{(33)}=1.9\right.$, $p=0.07)$; conscious: $3.33(\mathrm{SD}=0.61)$ vs $3.82(\mathrm{SD}=0.90$; $\left.\left.t_{(33)}=-1.26, p=0.22\right)\right]$, we decided not to remove these participants. Thirty-five participants were included in the main analyses (mean age, 25.9 years; $S D=5.9$ ).

\section{Experimental design}

Before the experiment, participants filled out the Vividness of Visual Imagery Questionnaire 2 (Marks, 1973), which is a 16 -item questionnaire that measures the general vividness of 
A

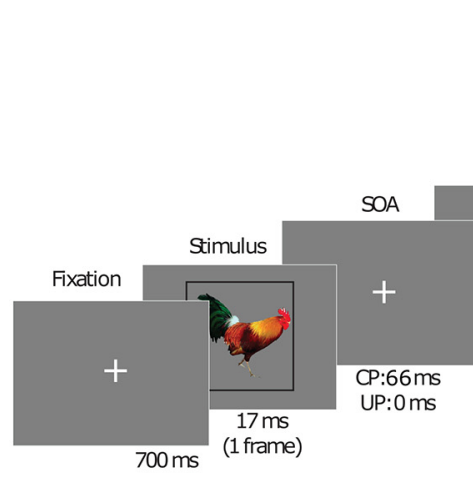

B

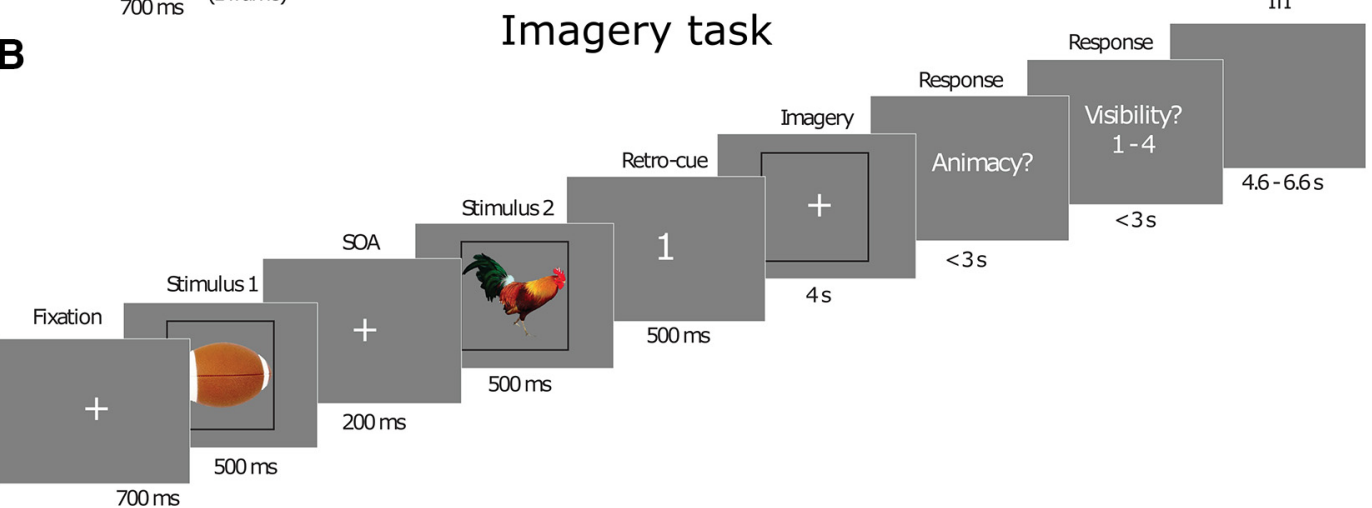

Masking task

III

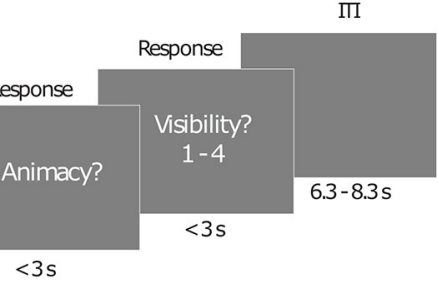

$600 \mathrm{~ms}$

$400 \mathrm{~ms}$

Imagery task
C Stimuli
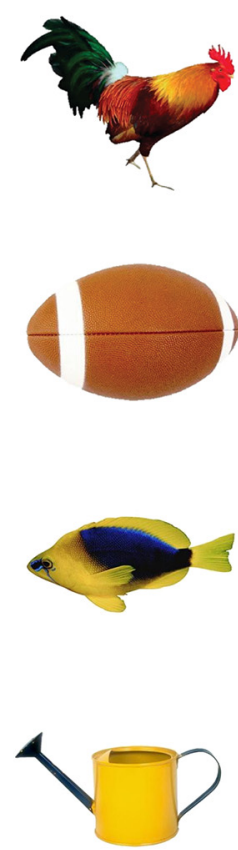

Figure 1. Experimental paradigm. A, Masking task. A stimulus is presented for $17 \mathrm{~ms}$, followed by a mask (duration, $400 \mathrm{~ms})$ after $0 \mathrm{~ms}$ (unconscious condition) or $66 \mathrm{~ms}$ (conscious condition). Participants had to indicate whether the stimulus was animate or inanimate and rate the visibility. $\boldsymbol{B}$, Visual imagery task. Participants were presented with two stimuli after each other followed by a cue indicating whether to imagine the first or the second stimulus, as vividly as possible. After the imagery, participants had to indicate whether the imagined stimulus was animate or inanimate and rate the visibility of their imagery. $\boldsymbol{C}$, Stimuli used: a rooster, a football, a fish, and a watering can from the POPORO stimulus dataset (Kovalenko et al., 2012). The neural analyses focused on pairwise comparisons between all possible combinations of stimuli.

a participant's imagery. The experiment consisted of two tasks, a perception and an imagery task, which were executed in interleaved blocks, and whether participants started with the imagery or perception task was counterbalanced over participants. The perception task consisted of conscious and unconscious trials, which only differed in ISI between the stimulus and the mask: $0 \mathrm{~ms}$ for the unconscious trials and $66 \mathrm{~ms}$ (four frames) for the conscious condition. We chose to operationalize conscious versus unconscious processing via experimental manipulation (strong vs weak masking) and not via post hoc trial selection based on visibility reports, because this latter approach has been shown to violate statistical assumptions and may lead to spurious unconscious effects (for more details, see Shanks, 2017). During the perception task, a stimulus was presented very briefly (17 ms), followed by a backward mask. Participants subsequently indicated whether the presented stimulus was animate or inanimate and rated the visibility of the stimulus on a scale from 1 (not visible at all) to 4 (perfectly clear; Fig. 1A). To prevent motor preparation, the response mapping for both the animacy and visibility ratings were randomized over trials. During the imagery task, two stimuli were each successively presented for $500 \mathrm{~ms}$, followed by a retro-cue indicating which of the two the participant should imagine. The participant then imagined the cued stimulus and subsequently indicated the animacy and the visibility of the imagined stimulus (Fig. 1B). The buttonresponse mapping for the animacy task and the visibility rating was randomized over trials to prevent motor preparation.

There were 184 conscious and 184 unconscious trials, and 46 repetitions per stimulus, divided over four blocks. Each conscious-unconscious block lasted $\sim 9 \mathrm{~min}$. There were 144 imagery trials, and 36 repetitions per stimulus, divided over four blocks. Each imagery block lasted $\sim 7 \mathrm{~min}$. The order of the different stimuli and SOAs (unconscious vs conscious trials) within the perception task and the stimuli and retro-cue combinations during imagery was fully counterbalanced within participants, and which task (imagery or perception) was executed first was randomized between participants. In total, there were eight blocks, leading to an experimental time of $\sim 65 \mathrm{~min} /$ participant. Including breaks and an anatomic scan, this added up to 90 min of fMRI scanning time.

\section{Stimuli}

We used stimuli from the POPORO (pool of pairs of related objects) stimulus dataset (Kovalenko et al., 2012), which contains color images of everyday objects and animals. From these stimuli, we selected four (two animate and two inanimate) for the final study. The stimuli were 
selected based on (1) familiarity and visual difference, such as to maximize classification performance; and (2) accuracy and visibility scores calculated in a pilot experiment. The stimuli were presented at $50 \%$ contrast on a gray background screen. They encompassed a $4 \times 4 \mathrm{~cm}$ square, which corresponded to a visual angle of $2.81^{\circ}$. The stimuli were relatively small to prevent large eye movements, which would affect our fMRI analyses. The mask was created by randomly scrambling the pixel values of all stimuli together and was also $4 \times 4 \mathrm{~cm}$ to fully mask the presented stimuli.

\section{Behavioral analysis}

To characterize performance on the discrimination animacy task we calculated $d^{\prime}$ as the distance between the signal and the signal plus noise, calculated as the difference between the hit rate and the false alarm rate (Macmillan and Creelman, 1990). A high $d^{\prime}$ value indicates better performance, and a $d^{\prime}$ value of zero indicates chance-level performance.

\section{fMRI acquisition}

Each block was scanned in a separate fMRI run, adding up to eight runs in total. In between runs, the researcher checked in with the participant and asked whether they needed a break. The experiment continued when the participant said they were ready to continue. $\mathrm{AMRI}$ data were recorded on a Siemens 3 T Skyra scanner with a Multiband 6 sequence (TR, $1 \mathrm{~s}$; voxel size, $2 \times 2 \times 2$ $\mathrm{mm}$; TE, $34 \mathrm{~ms}$ ) and a 32-channel head coil. For all participants, the field of view was tilted $-25^{\circ}$ from the transverse plane, using the Siemens AutoAlign Head software, resulting in the same tilt relative to the individual participant's head position. T1-weighted structural images (MPRAGE; voxel size, $1 \times 1 \times 1 \mathrm{~mm}$; TR, $2.3 \mathrm{~s}$ ) were also acquired for each participant.

\section{fMRI preprocessing}

Before decoding analyses, data were preprocessed using SPM12 (RRID:SCR_007037). All functional imaging data were motion corrected (realignment) and coregistered to the T1 structural scan. The scans were then normalized to $\mathrm{MNI}$ space using DARTEL (diffeomorphic anatomical registration through exponentiated lie algebra) normalization and smoothed with a $6 \mathrm{~mm}$ Gaussian kernel, which has been shown to improve group-level decoding accuracy (Misaki et al., 2013; Gardumi et al., 2016; Hendriks et al., 2017). A high-pass filter of $128 \mathrm{~s}$ was used to remove slow signal drift.

\section{Multivariate pattern analysis}

Multivariate analyses were performed using MATLAB version 2018a (RRID:SRC_001622). We used linear discriminant analysis to decode the stimulus identity per searchlight based on the $\beta$-estimates per trial. All individual trial $\beta$-estimates were obtained from one general linear model (GLM) that contained a separate regressor for each trial set at the onset of the image [or imagery frame for imagery with a duration of 0 (spike) for the conscious and unconscious conditions and a duration of 4 for the imagery condition; Bosch et al., 2014; Dijkstra et al., $2017 a, b]$. Additional regressors in this GLM were (1) the animacy response screen onsets, duration set to the time until response; (2) animacy response button presses, duration 0 (spike); (3) the visibility response screen onsets, duration set to the until response; (4) visibility response button presses, duration 0 (spike); (5) onset of the first stimulus in the retro-cue task, duration $500 \mathrm{~ms}$; (6) onset of the second stimulus in the retro-cue task, duration $500 \mathrm{~ms}$; and (7) a constant value per run to eliminate runspecific changes in mean signal amplitude. Finally, the average signals from the white matter and CSF (Lund et al., 2005; Caballero-Gaudes and Reynolds, 2017) as well as the motion parameters were included as nuisance regressors. Decoding within and across conditions was done pairwise between all combinations of the four stimuli, resulting in six decoding pairs, over which the accuracy was then averaged. Searchlights had a radius of four voxels, resulting in 257 voxels/searchlight on average. Searchlights moved through the brain based on the center voxel such that voxels participated in multiple searchlights (Allefeld and Haynes, 2014). Leave-onerun-out cross-validation was performed, such that for each fold, a classifier was trained on three runs and tested on the fourth, left-out run. This was done for all comparisons except for imagery-conscious and imagery-unconscious cross-decoding, because these data already came from different task runs (Fig. 1). Generalization across conditions is often asymmetric, for which there could be a variety of reasons such as differences in signal-to-noise ratio between the two conditions (van den Hurck and Op de Beeck, 2019). Because we did not have a priori hypotheses about asymmetries in cross-decoding directions and because both directions revealed qualitatively similar results, we average across both cross-decoding directions before doing statistics across subjects. The names of the regions containing stimulus specific information were determined using the AAL AAL (Automated Anatomical Labeling) atlas (Tzourio-Mazoyer et al., 2002).

\section{Psychophysiological interaction analysis}

After identifying a visual area that contained stimulus information (significant stimulus decoding) in all three conditions, we performed a psychophysiological interaction (PPI) analysis to investigate differences in connectivity between this area and the rest of the brain between the conditions (Friston et al., 1997). Per participant, the seed region was defined as an $8 \mathrm{~mm}$ sphere centered on the peak averaged univariate activation over the three conditions, within a $16 \mathrm{~mm}$ sphere centered around the voxels in which decoding was significant for all three conditions at the group level (see Fig. 3; MNI coordinates: $-54,-65$, $-10)$. This approach ensures that approximately the same region was used for every participant while also taking account of differences in structural and functional anatomy between participants. This method and the size of region of interest (ROI) definition are based on recommendations in the literature for comparable analyses (Zeidman et al., 2019). One participant was excluded because the $t$ 
contrast of the averaged activation over the three conditions versus 0 did not reach the statistical threshold of 0.05 in any of the voxels within the group sphere. The following two PPI contrasts were calculated: (conscious perception + unconscious processing) $>$ imagery (feedforward); and (conscious perception + imagery) $>$ unconscious processing (feedback). Connectivity with significant areas was compared in a post hoc analysis by calculating the difference in connectivity between each two conditions (see Fig. 4C; Friston et al., 1997). Note that the connectivity analyses were not stimulus specific; therefore, the first comparison, where we compare conditions that contained a mask (conscious + unconscious) with conditions that did not contain a mask (imagery), might be driven (partly) by processing of the mask instead of the stimuli preceding the mask.

\section{Statistical analysis}

The application of standard second-level statistics, including $t$ tests, to multivariate pattern analysis (MVPA) measures is in many cases invalid because of violations of assumptions. Therefore, we used permutation testing to generate the empirical null distribution, thereby circumventing the need to rely on assumptions about this distribution. We followed the approach suggested by Stelzer et al. (2013) for searchlight MVPA measurements, which uses a combination of permutation testing and bootstrapping to generate chance distributions for group studies. Because of the large computational load of searchlight decoding analysis, per participant, 25 permutation maps were generated by permuting the class labels within each run. Group-level permutation distributions were subsequently generated by bootstrapping over these 25 maps (i.e., randomly selecting one of 25 maps per participant and then averaging over participants. A total of 10,000 bootstrapping samples were used to generate the group null distribution per voxel and per comparison. The $p$ values were calculated per voxel as the right-tailed area of the histogram of permutated accuracies from the mean over participants. We corrected for multiple comparisons using whole-brain false discovery rate correction with a $q$ value cutoff of 0.01 . Cluster correction was performed, ensuring that voxels were only identified as being significant if they belonged to a cluster of at least 50 significant voxels (Dijkstra et al., 2017a).

\section{Data availability}

All data will be made publicly available on publication of this article. Analysis code for this study will be made available via the corresponding author on request.

\section{Results}

\section{Behavioral results}

To check whether participants indeed did not consciously perceive the stimuli in the unconscious condition, we tested their perceptual sensitivity and visibility scores. Whereas the value of $d^{\prime}$ was clearly significantly above zero for both the conscious (mean $=3.74, \mathrm{SD}=0.87$; $\left.t_{(34)}=25.40, p<0.0001\right)$ as well as the imagery (mean $\left.=3.32, \mathrm{SD}=0.83 ; t_{(34)}=23.74, p<0.0001\right)$ conditions, this was not the case for the unconscious condition (mean $=0.05, \mathrm{SD}=0.20 ; t_{(34)}=1.57, p=0.127 ; \mathrm{BF} 01=0.549 ; \mathrm{Fig}$. $2 A)$. Furthermore, the $d^{\prime}$ value was significantly higher for both the conscious condition $\left(t_{(34)}=23.18, p<0.0001\right)$ and the imagery condition $\left(t_{(34)}=20.60, p<0.0001\right)$ compared with the unconscious condition. The $d^{\prime \prime}$ value in the conscious condition was also slightly higher than in the imagery condition $\left(t_{(34)}=2.62, p=0.013\right)$. Furthermore, the visibility ratings for both the conscious condition (mean $=3.03, \mathrm{SD}=0.54$; $\left.t_{(34)}=10.94, p<0.0001\right)$ as well as the imagery condition (mean $=2.91, \mathrm{SD}=0.38 ; t_{(34)}=11.76, p<0.0001$ ) were much higher than for the unconscious condition (mean $=1.37$, $\mathrm{SD}=0.54$; Fig. $2 B$ ). A few participants rated a proportion of trials in the unconscious condition as high visibility (Fig. 2B); however, all of these participants still had a discrimination accuracy at chance (all $<53.3 \%)$. Furthermore, there was no significant relationship between the mean visibility rating and $d^{\prime}$ in the unconscious condition over participants $(r=0.14$, $p=0.41$ ). Given the nonsignificant task performance and the potential confusion caused by the randomization of response mapping between trials, these high visibility ratings during the unconscious condition are unlikely to reflect true conscious visibility. Together, these results suggest that the stimuli were indeed strongly masked, and therefore we were able to isolate feedforward processing as much as possible (Fahrenfort et al., 2007).

\section{Decoding within conditions}

To investigate which areas represented stimulus information during the three conditions, we performed a searchlight decoding analysis separately for each condition (Fig. 3). Statistical tests were performed using grouplevel permutation testing as described in the study by Stelzer et al. (2013) and corrected for multiple comparisons (see Materials and Methods). Significant decoding clusters are shown in Figure 3 and are listed in Table 1. The cutoff accuracy value for significance was 0.508 for the unconscious and conscious conditions, and 0.511 for imagery. The relatively low decoding accuracy of conscious representations $(\sim 0.55)$ compared with other studies ( 0.55 to 0.65; Eger et al., 2008; Axelrod and Yovel, 2015) is likely because of the backward mask, which adds noise to the stimulus response. Given the low temporal resolution of $\mathrm{FMRI}$, this means that the BOLD signal at the time of the stimulus will contain a mixture of stimulus response and response to the mask, increasing variance unrelated to the stimulus and thereby decrease decoding performance. In line with previous studies (Pearson et al., 2015; Dijkstra et al., 2019), we could decode stimulus information during conscious perception as well as imagery in low- and high-level visual areas, intraparietal sulcus and lateral frontal cortex (Fig. 3B-E). Interestingly, significant decoding of unconscious stimuli was observed only in left highlevel visual cortex, temporal pole, and lateral frontal cortex (Fig. $3 A$ ). There was no significant unconscious decoding in low-level visual areas. All three conditions showed stimulus representations in left LOC (Fig. 3E).

\section{Psychophysiological interaction analysis}

The decoding analysis showed that left LOC contained stimulus information during all three conditions (Fig. $3 E$, 
A

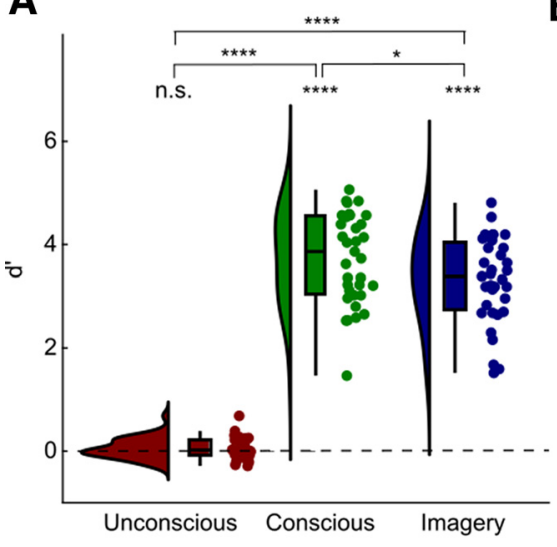

B

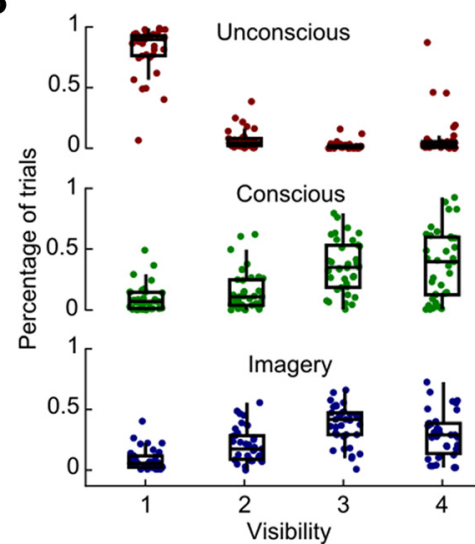

Figure 2. Behavioral results. A, The $d^{\prime}$ values for the animacy task are shown separately for each condition. The bell-shaped curves represent the distribution over participants, the boxplots indicate the four quartiles, and the dots represent individual participants. The $d^{\prime}$ value was significantly higher than zero in the conscious as well as imagery condition, but not in the unconscious condition. ${ }^{*} p<0.05,{ }^{* \star \star *} p<0.0001$. B , Percentage of trials of each visibility rating (1-4) separately for the three conditions. Boxplots represent the distributions over participants, and dots represent individual participants.

lateral view), suggesting that this area might be where feedback and feedforward signals overlap. Before directly investigating the representational overlap between conditions using across-condition decoding generalization, we first investigated whether this area indeed showed more feedback connectivity during conscious perception and imagery compared with unconscious processing and more feedforward connectivity during conscious and
A

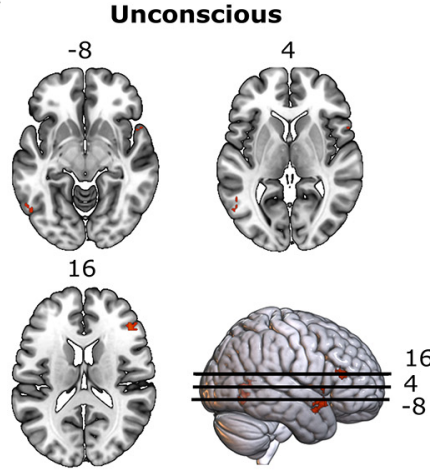

D Unique within-decoding
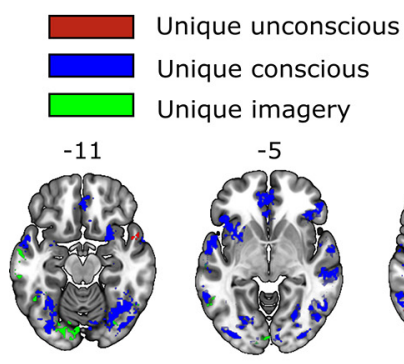

B

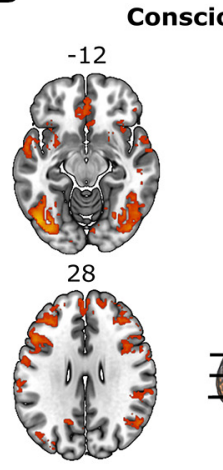

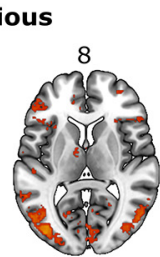

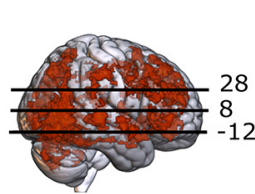

Decoding accuracy

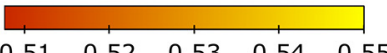

C

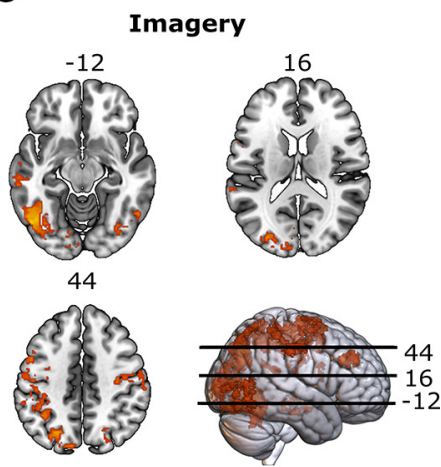

\section{E Spatial overlap within-decoding}
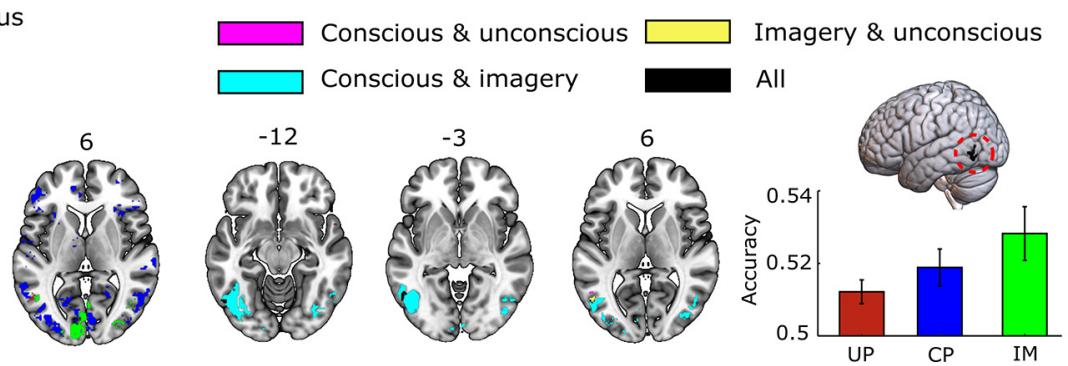

Figure 3. Condition-specific neural representations. $\boldsymbol{A}-\boldsymbol{C}$, For each condition, significant decoding clusters are shown for various axial slices. The heatmap indicates average decoding accuracy. $\boldsymbol{D}, \boldsymbol{E}$, Significant decoding accuracy clusters are unique for each condition $(\boldsymbol{D})$ and for spatial overlapping between conditions (E). Significant decoding accuracy was found in all three conditions (indicated in black, circled in red) around the left LOC at the following MNI coordinates: $-54,-65,-10$. Decoding accuracies for the three conditions (UP, unconscious processing; CP, conscious perception; IM, imagery) within this $\mathrm{ROI}$ are plotted, with the error bars indicating the standard error of the mean (SEM). 
Table 1: Significant within decoding clusters

\begin{tabular}{|c|c|c|c|c|c|c|c|}
\hline \multirow[b]{2}{*}{ Lobe } & \multirow[b]{2}{*}{ Atlas label } & \multicolumn{5}{|c|}{ MNI peak } & \multirow[b]{2}{*}{ Peak accuracy } \\
\hline & & Condition & $x$ & $y$ & $z$ & Voxels, $N$ & \\
\hline \multirow{4}{*}{ Occipital } & Occipital_Inf_L & Conscious & -48 & -70 & -6 & 9302 & 0.54 \\
\hline & Occipital_Inf_R & Imagery & 46 & -76 & -2 & 459 & 0.53 \\
\hline & Cuneus_L & Conscious & 0 & -72 & 34 & 171 & 0.52 \\
\hline & Calcarine_R & Conscious & 12 & -60 & 14 & 115 & 0.52 \\
\hline \multirow{5}{*}{ Temporal } & & & 64 & -2 & -10 & 220 & 0.52 \\
\hline & Temporal_Sup_L & Imagery & -64 & -38 & 20 & 100 & 0.53 \\
\hline & Temporal_Mid_L & Imagery & -60 & -20 & -20 & 182 & 0.53 \\
\hline & Temporal_Inf_L- & Unconscious & -56 & -62 & -6 & 86 & 0.52 \\
\hline & Temporal_Pole_Sup_R & Unconscious & 52 & 14 & -12 & 91 & 0.52 \\
\hline \multirow[t]{4}{*}{ Parietal } & Parietal_Inf_L & Conscious & -32 & -36 & 40 & 72 & 0.52 \\
\hline & & Imagery & 64 & -22 & 40 & 90 & 0.52 \\
\hline & Cingulum_Mid_L & Imagery & -4 & 30 & 32 & 263 & 0.53 \\
\hline & Cingulum_Mid_R & Conscious & 8 & -34 & 42 & 56 & 0.52 \\
\hline \multirow[t]{9}{*}{ Frontal } & Frontal_Sup_Medial_L & Conscious & -6 & 58 & 22 & 468 & 0.52 \\
\hline & Frontal_Sup_R & Conscious & 18 & 52 & 26 & 91 & 0.52 \\
\hline & & Imagery & 24 & -4 & 60 & 172 & 0.53 \\
\hline & Frontal_Inf_Tri_L & Conscious & -48 & 18 & 28 & 1738 & 0.53 \\
\hline & & Unconscious & 44 & 36 & 16 & 62 & 0.52 \\
\hline & Frontal_Med_Orb_R & Conscious & 2 & 46 & -4 & 575 & 0.52 \\
\hline & Supp_Motor_Area_L & Imagery & -6 & 4 & 68 & 557 & 0.63 \\
\hline & Precentral_L & Conscious & -56 & -2 & 26 & 76 & 0.52 \\
\hline & & Imagery & -56 & 8 & 26 & 59 & 0.52 \\
\hline Cerebellum & Cerebellum_Crus2_R & Conscious & 30 & -80 & -40 & 71 & 0.52 \\
\hline
\end{tabular}

Atlas labels were determined using the AAL (Automated Anatomical Labeling) atlas (Tzourio-Mazoyer et al., 2002) on the basis of the MNI coordinates of the peak decoding accuracy.

unconscious processing compared with imagery. To investigate this, we performed a PPI analysis to characterize differences in brain connectivity among the three conditions (Fig. 4, Table 2).

In line with the predictions, there was stronger connectivity during conscious perception and unconscious processing compared with imagery between left LOC and EVC (MNI coordinates: $-1,-85,9)$ as well as right LGN (MNI coordinates: 24, -29 , 4; Fig. $4 A-C$ ), in line with the idea that during these conditions there was more feedforward processing than during imagery. However, because these conditions also differed in whether a mask was presented (conscious and unconscious) or not (imagery), and the PPI analysis is not stimulus specific, this feedforward connectivity might partly reflect processing of the mask and not the (unconscious) stimulus before the mask. Furthermore, there was stronger connectivity during conscious perception and imagery compared with unconscious processing between left LOC and bilateral dorsolateral prefrontal cortex (dIPFC; left MNI coordinates: $-45,36$, 9; right $\mathrm{MNI}$ coordinates: 48, 36. 9) and right lateral frontal cortex, in line with increased feedback connectivity during these conditions. Post hoc direct comparisons between conditions of the regions showing significant changes in connectivity (Fig. 4A,B) showed that connectivity between EVC and left LOC was stronger during conscious perception compared with imagery as well as during unconscious processing compared with imagery (Fig. 4C, left). Furthermore, coupling between left LOC and left dIPFC was stronger during conscious perception compared with unconscious processing as well as during imagery compared with both conscious and unconscious processing (Fig. 4C, middle). Finally, coupling between left LOC and right dIPFC was stronger during imagery compared with both conscious and unconscious processing (Fig. 4C, right). These results indicate that, in line with our assumption, long-range feedback processing is indeed stronger during conscious perception and imagery compared with unconscious processing.

\section{Generalization across conditions}

The above decoding analysis showed that left LOC contained stimulus information during all three conditions (Fig. 3E, lateral view), suggesting that this area might be where feedback and feedforward signals overlap. To directly test whether the representations between conditions were similar, we performed across-condition decoding, where we trained a classifier to dissociate the stimuli in one condition and tested it in another 

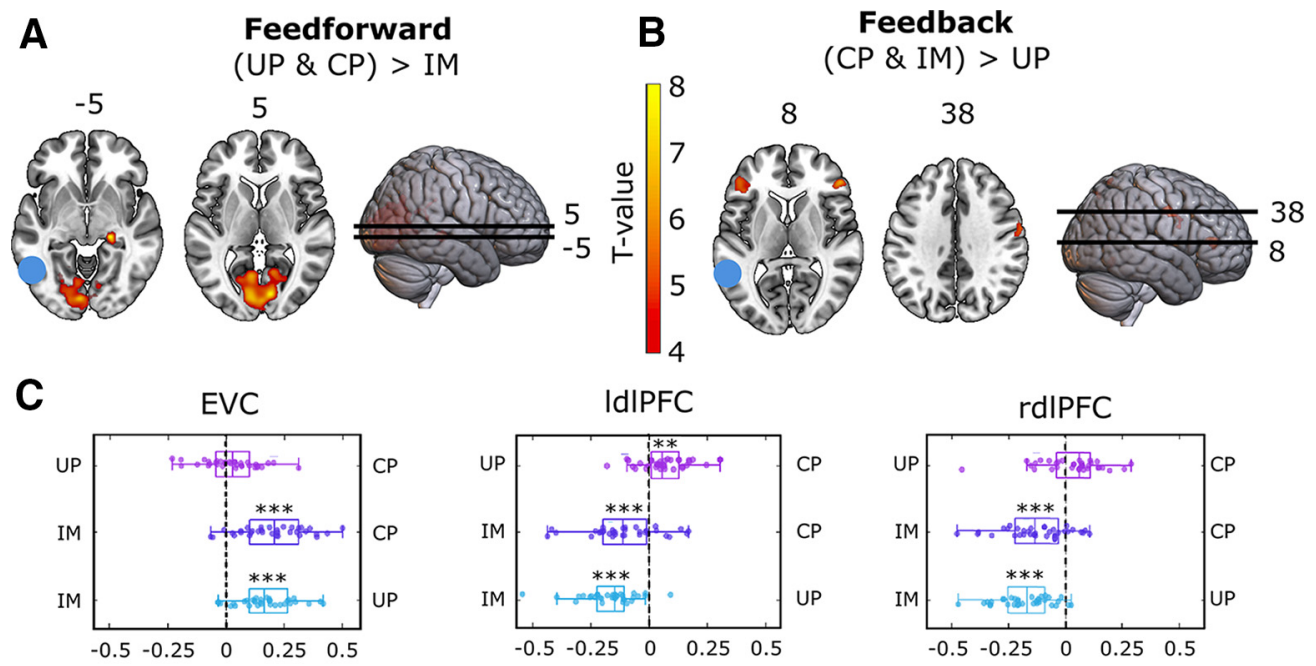

Difference in connectivity strength

Figure 4. Psychophysiological interactions with left LOC as the seed region. $\boldsymbol{A}$, The blue dot illustrates the location of the seed region, red-yellow indicates brain areas that showed significantly stronger connectivity with left LOC during conscious perception (CP) and unconscious processing (UP) compared with imagery (IM; i.e., in conditions where feedforward connections were present vs in those where they were not). $\boldsymbol{B}$. The blue dot indicates the location of the seed region, red-yellow indicates brain areas that showed significantly stronger connectivity with left LOC during conscious perception and imagery compared with unconscious processing (i.e., in conditions where feedback connections were present vs in those where they were not). $\boldsymbol{C}$, Direct comparisons of connectivity between all conditions for left high-level visual cortex and EVC (left); left high-level visual cortex and left dIPFC (IdIPFC; middle); and left high-level visual cortex and right dIPFC (rdIPFC). Boxplots indicate variance over participants, and dots represent individual participants. ${ }^{* *} p<0.005,{ }^{* * *} p<0.0005$.

condition. In this analysis, above-chance cross-decoding accuracy would indicate that the underlying stimulus representations are to some extent similar. Significant across-condition clusters are shown in Figure 5 and are listed in Table 3. In line with previous studies (Reddy et al., 2010; Lee et al., 2012; Pearson and Kosslyn, 2015; Dijkstra et al., 2017a, 2019), we found representational overlap between conscious perception and imagery in visual, parietal, and frontal areas (Fig. 5A, Table 1). In contrast, there was no significant cross-decoding between the unconscious condition and the other conditions in any brain area, suggesting an absence of representational overlap. Furthermore, despite the significant decoding in left LOC within all conditions (unconscious: mean $=0.512, \mathrm{SD}=0.063$; conscious: mean $=0.519$,
$\mathrm{SD}=0.097$; imagery: mean $=0.528, \mathrm{SD}=0.098)$, there was no significant cross-decoding overlap between the unconscious condition and the other conditions in this area, even at lower statistical thresholds (Fig. 5B).

Together, these results suggest that there is no representational overlap between unconscious and imagined neural representations. However, it is possible that we did not observe significant representational overlap here, not because there is no overlap, but because we do not have enough power to reveal this overlap. The results presented in Figure $5 B$ show that cross-decoding accuracy between conscious perception and imagery is significantly higher than the cross-decoding accuracy between the other conditions. This means that while we cannot exclude the possibility of overlap with unconscious

Table 2: Clusters connected with high-level within-decoding spatial overlap cluster

\begin{tabular}{|c|c|c|c|c|c|c|c|}
\hline \multirow[b]{2}{*}{ Lobe } & \multirow[b]{2}{*}{ Atlas label } & \multicolumn{4}{|c|}{ MNI peak } & \multirow[b]{2}{*}{ Voxels, $N$} & \multirow[b]{2}{*}{ Peak $t$ value } \\
\hline & & Comparison & $x$ & $y$ & $z$ & & \\
\hline Temporal & Temporal_Inf_L & $(\mathrm{CP}$ and $\mathrm{IM})>\mathrm{UP}$ & -54 & -58 & -8 & 87 & 5.11 \\
\hline \multirow{3}{*}{ Parietal } & Parietal_Sup_R & $(\mathrm{CP}$ and $\mathrm{IM})>\mathrm{UP}$ & 16 & -60 & 68 & 62 & 5.73 \\
\hline & Precuneus_L & $(\mathrm{CP}$ and $\mathrm{UP})>\mathrm{IM}$ & -10 & -52 & 20 & 53 & 4.6 \\
\hline & Postcentral_R & $(\mathrm{CP}$ and $\mathrm{IM})>\mathrm{UP}$ & 62 & -4 & 36 & 120 & 5.63 \\
\hline Frontal & Frontal_Inf_Oper_R & $(\mathrm{CP}$ and $\mathrm{IM})>\mathrm{UP}$ & 48 & 4 & 22 & 60 & 4.66 \\
\hline Other & Lateral Gen Nuc & $(\mathrm{CP}$ and $\mathrm{UP})>\mathrm{IM}$ & 22 & -28 & -4 & 80 & 9.12 \\
\hline
\end{tabular}

Atlas labels determined using the AAL (Automated Anatomical Labeling) atlas (Tzourio-Mazoyer et al., 2002) on the basis of the MNI coordinates of the peak $t$ value for the PPI analysis. CP, Conscious perception; UP, unconscious processing; IM, imagery. 
A

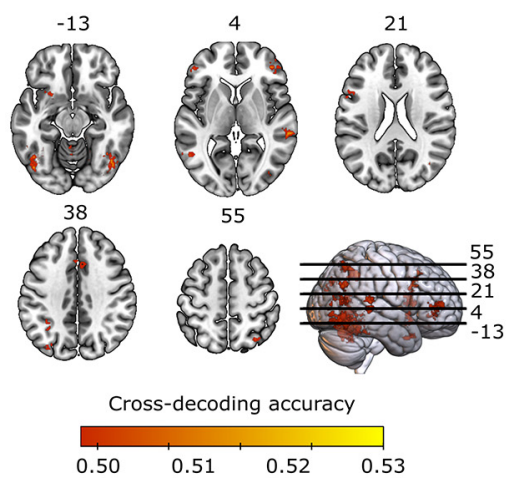

B

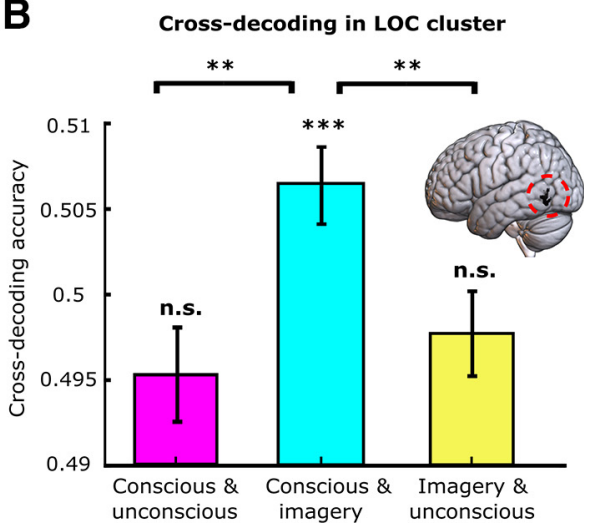

Figure 5. Across condition decoding accuracy. There was only significant representational overlap between conscious perception and mental imagery. $\boldsymbol{A}$, Significant cross-decoding clusters are shown for various axial slices. $\boldsymbol{B}$, Cross-decoding accuracy within the LOC cluster that had significant within-condition decoding in all three conditions (Fig. $3 E$ ), the same voxels were evaluated in all comparisons. Error bars indicate the SEM. n.s., Nonsignificant. ${ }^{*} p<0.05,{ }^{\star \star} p<0.01,{ }^{\star * *} p<0.005$

representations, we can conclude that representational overlap with unconscious representations is lower than the overlap between conscious and imagined representations. However, this might partly be because of the fact that unconscious representations were less strong compared with the other conditions (Fig. 3). We discuss this possibility in more detail in the Discussion.

\section{Discussion}

In this study, we aimed to investigate the overlap between neural representations caused by feedforward versus feedback signals by comparing brain activity during mental imagery, conscious perception and unconscious processing. We found significant stimulus decoding for all three conditions in left high-level visual cortex (i.e., LOC). Furthermore, a PPI analysis showed that this area indeed showed more feedback connectivity during conscious perception and imagery compared with unconscious processing. These results suggested that this area might be the place where feedforward and feedback-initiated representations overlap. However, across-condition generalization revealed there was only significant representational overlap in this area between conscious perception and imagery, but not unconscious perception. These findings are in line with the idea that feedback changes the "format" of neural representations, leading to the reduction of overlap between representations caused by feedforward and feedback signals, but the presence of overlap between representations caused by feedback processes associated with perception of external stimuli and feedback processes associated with mental imagery.

The significant decoding of unconscious category-specific stimuli in high-level cortex agrees with previous findings (Jiang and He, 2006; Rees, 2007; Fahrenfort et al., 2012; Axelrod et al., 2015). Although both conscious and unconscious category-specific representations were present in high-level visual cortex, we did not find representational overlap between the two. This is in line with

Table 3: Significant across condition decoding clusters

\begin{tabular}{|c|c|c|c|c|c|c|}
\hline \multirow[b]{2}{*}{ Lobe } & \multicolumn{4}{|c|}{ MNI peak } & \multirow[b]{2}{*}{ Voxels, $N$} & \multirow[b]{2}{*}{ Peak accuracy } \\
\hline & Atlas label & $x$ & $y$ & $z$ & & \\
\hline Occipital & Occipital_Inf_R & 44 & -78 & -4 & 261 & 0.52 \\
\hline \multirow[t]{3}{*}{ Temporal } & Temporal_Mid_R & 60 & -34 & 4 & 122 & 0.52 \\
\hline & Temporal_Pole_Sup_L & -46 & 16 & -26 & 72 & 0.51 \\
\hline & Fusiform_L & -46 & -64 & -18 & 641 & 0.52 \\
\hline & Cingulum_̄Mid_R & 4 & 14 & 30 & 79 & 0.52 \\
\hline & Precuneus_L & -16 & -56 & 14 & 76 & 0.52 \\
\hline & Angular_R & 48 & -62 & 32 & 60 & 0.51 \\
\hline \multirow[t]{3}{*}{ Frontal } & Frontal_Sup_Orb_L & -26 & 14 & -14 & 59 & 0.52 \\
\hline & Frontal_Mid_R & 46 & 52 & 8 & 113 & 0.52 \\
\hline & Frontal_Inf_Oper_L & -50 & 12 & 12 & 183 & 0.52 \\
\hline
\end{tabular}

Atlas labels determined using the AAL (Automated Anatomical Labeling) atlas (Tzourio-Mazoyer et al., 2002) on the basis of the MNI coordinates of the peak decoding accuracy. Condition is not indicated here because only imagery-conscious across-condition decoding was significant. 
previous studies using backward masking (Bar et al., 2001) and dichoptic fusion (Schurger et al., 2010). These studies also showed conscious and unconscious representations in high-level visual cortex, but no spatial or representational overlap between them. Conscious and unconscious representations may differ in several respects, including their duration, intensity, coherence, stability, and reproducibility (Lamme and Roelfsema, 2000; Tononi and Koch, 2008; Schurger et al., 2010, 2015). It has been proposed that long-range feedback may stabilize activity in local neural processors, as if the brain "decides" what specific input it has received. The decision of the network, given the input, is what may be reflected in conscious access (Dehaene, 2014; Schurger et al., 2015). The stabilization of neural activity by feedback therefore may change the format of neural category-specific representations (Dehaene et al., 2003; King et al., 2016; Baria et al., 2017; Dijkstra et al., 2018; He, 2018; Weaver et al., 2019; Xie et al., 2020).

Although an intriguing possibility, some previous fMRI studies did report cross-decoding between conscious and unconscious conditions (Moutoussis and Zeki, 2002; Sterzer et al., 2008; Sterzer and Rees, 2008; Fahrenfort et al., 2012). In these studies, awareness of face/house stimuli was manipulated by dichoptic fusion (Moutoussis and Zeki, 2002; Fahrenfort et al., 2012), continuous flash suppression (CFS; Sterzer et al., 2008), or binocular rivalry (Sterzer and Rees, 2008). Which specific brain areas retain information about unconscious stimuli likely depends on the methods used to render the stimuli invisible (Fogelson et al., 2014; Izatt et al., 2014; Axelrod et al., 2015). Dichoptic fusion, CFS, and binocular rivalry all rely on interactions between inputs from the two eyes and may primarily affect inhibition-adaptation cycles as early as V1, although much is still unclear at present (Tong et al., 2006; Rees, 2007; Axelrod et al., 2015). In contrast, the neural effects of backward masking have previously been shown to disrupt recurrent interactions between high- and low-level visual regions (Lamme et al., 2002; Roelfsema et al., 2002; Del Cul et al., 2007; Fahrenfort et al., 2007; van Gaal and Lamme, 2012). Future research is necessary to fully determine the specific effects of each visibility manipulation on neural processing to unravel the discrepancies between studies and to understand why representational overlap between conscious and unconscious representations is sometimes observed and sometimes not.

The idea that feedback processing changes the format of neural representations suggests that the representational overlap between these different modes of perception should change over time. Because of the sluggishness of the BOLD response, fMRI lacks the temporal resolution needed to characterize such dynamics. In contrast, recent studies using methods with higher temporal resolution such as electroencephalography and magnetoencephalography (MEG) do indeed suggest differences in the timing of representational overlap among conscious perception, unconscious processing, and imagery. During conscious perception, neural representations first change rapidly over time during early time windows, likely reflecting the feedforward sweep, after which representations stabilize later in time, presumably via recurrent processing (Cichy et al., 2014; Mostert et al., 2015; Schurger et al., 2015; Baria et al., 2017; Dijkstra et al., 2018; He, 2018). Recent evidence shows that neural representations of stimuli that were strongly masked or missed during the attentional blink, only overlap with conscious conditions at early stages of input processing (until 250 ms; Meijs et al., 2019; Weaver et al., 2019). Furthermore, a recent MEG study revealed that representations during imagery mostly overlap with representations during later stages of conscious perception (Dijkstra et al., 2018; Xie et al., 2020). This supports the idea that neural representations of consciously reported and unreported stimuli are similar during initial feedforward (and likely local recurrent) processing, but that long-range feedback changes the neural representations, which then mimics the representations initiated by imagery-related feedback processing.

It is important to note that the exact relationship between (long-range) feedback processing and conscious awareness is still debated (Boly et al., 2017). Some theories suggest that local recurrent processing within sensory systems is sufficient for conscious experience (Lamme, 2015), whereas others propose that communication within a broader network, including frontoparietal areas, is necessary (Dehaene and Changeux, 2011; Mashour et al., 2020) and still others propose that activation of meta-representations is sufficient (Brown et al., 2019; Lau and Rosenthal, 2011). Here, we used perception rendered unconscious via backward masking as a proxy for feedforward visual processing, and in line with this assumption, our PPI results suggested that visual activity was only driven in a feedforward fashion in the unconscious condition. However, it is possible that there was still some form of feedback processing present during the unconscious condition, either weaker or more local compared with the conscious condition, that was not picked up by the PPI analysis. This means that the absence of representational overlap between the conscious and unconscious conditions might be because of other factors that are affected by awareness in addition to feedback processing. Future research directly investigating how top-down processing changes neural representations, using methods with a higher temporal resolution, will give more insight into this issue.

Finally, in line with previous studies we not only found significant cross decoding between conscious perception and imagery in several visual areas (Reddy et al., 2010; Cichy et al., 2012; Lee et al., 2012; O'Craven and Kanwisher, 2000; Albers et al., 2013; Dijkstra et al., 2017a, b), but also in parietal and frontal areas (Christophel et al., 2017; Dijkstra et al., 2017a,b). Additionally, we observed stronger connectivity between LOC and the dIPFC during imagery and conscious perception than during unconscious perception. The dIPFC has been implicated in numerous studies investigating the neural mechanisms of conscious reportability (conscious access) of input (Dehaene et al., 2006; Lau and Passingham, 2006; Rees, 2007; Davidson et 
al., 2010). These studies, in a similar way to ours, have all focused on conscious access of an external stimulus, whereas a recent study showed similar feedback connectivity during conscious perception and mental imagery (Dijkstra et al., 2017b). The current results suggest that dIPFC is important for conscious access, regardless of whether it is internally or externally generated. However, it should be noted that our perception task was not passive; participants actively attended to specific features of the stimulus to judge its animacy. Therefore, the overlap between imagery and perception reported here might (partly) be because of the use of similar attentional mechanisms (Dijkstra et al., 2019). During both the perception and imagery tasks, participants had to attend to specific spatial locations and features to correctly execute the animacy task. This means that during both tasks, spatial and feature-based top-down attention was used. Moreover, the increase in dIPFC connectivity during imagery compared with conscious perception might reflect the increased attentional load of generating a sensory representation in the absence of its corresponding input (Dijkstra et al., 2017a,b). Furthermore, the nature of the imagery task used here, in which the imagined image is presented relatively shortly before the imagery, might result in lingering feedforward activity. Several studies using the same paradigm only showed feedback processing during imagery (Dijkstra et al., 2017b, 2020); however, we cannot completely rule out that the imagery also contained some feedforward processing. To fully address this, future research should investigate whether similar patterns are found with conscious but passive perception and with imagery initiated from long-term memory.

An alternative possibility for our findings is that feedback does not change the representational format per se, but that during the conscious condition, feedback enhances representations of feedforward information, for example, via gain increase (Reynolds and Heeger, 2009; Wyart et al., 2012). Our results would then suggest that this kind of feedback-related enhancement is necessary to detect representational overlap between perception and imagery. This would also mean that using more sensitive methods, such as single-cell recordings, might still uncover representational overlap between the neural populations recruited during imagery and those activated by unconsciously processed stimuli.

Related to this, it is important to note that while we did find significant decoding within unconscious processing, the decoding accuracy in this condition was lower than during both imagery and conscious perception. This means that our power to detect representational overlap with the unconscious condition was lower compared with the other conditions. Therefore, we cannot rule out that our lack of representational overlap with unconscious processing is because of low unconscious decoding. It is theoretically possible that the amount of representational overlap with unconscious conditions is as high as the other conditions, but that the low power within the unconscious condition prevented us from detecting this. Low unconscious decoding may partly reflect an inherent feature of unconscious processes, in the sense that feedforward initiated representations are less strong (especially higher up in the cortical hierarchy) compared with representations that have been stabilized via long-range feedback connections as mentioned above (Lamme and Roelfsema, 2000; Tononi and Koch, 2008; Schurger et al., 2010, 2015), leading to lower decoding accuracy and therefore less power to detect representational overlap (Fahrenfort et al., 2012; van Gaal and Lamme, 2012; Weaver et al., 2019). Furthermore, although this type of masking has been shown to selectively disrupt feedback processing while keeping feedforward activity intact (Fahrenfort et al., 2007; van Gaal et al., 2008, 2011), because of the low temporal resolution of the BOLD signal we are unable to completely rule out a reduction in feedforward activity because of the masking procedure. To fully rule out this possibility, ideally, the within-decoding accuracy in all conditions is equalized experimentally, for example by lowering the contrast of the stimulus in the conscious condition (for a similar approach in behavior, see Lau and Passingham, 2006). This is an interesting avenue for future research.

In summary, our results show that neural representations measured by fMRI, triggered by purely feedforward (unconscious processing) or feedback (mental imagery) processes show reduced overlap. This suggests that the large representational overlap between imagery and perception reported in the literature (Dijkstra et al., 2019; Pearson, 2019) is undetectable for stimulus-triggered activation in the absence of feedback processing. Our results suggest that long-range feedback processing alters the format or strength of neural representations, for example, through stabilization of the neural code. More insight into this dynamic process can be gained using methods with higher temporal resolution than fMRI. Future research should explore exactly how feedback changes the format of representations and how different methods of rendering stimuli invisible affect this process.

\section{References}

Albers AM, Kok P, Toni I, Dijkerman HC, de Lange FP (2013) Shared representations for working memory and mental imagery in early visual cortex. Curr Biol 23:1427-1431.

Allefeld C, Haynes JD (2014) Searchlight-based multi-voxel pattern analysis of $\mathrm{fMRI}$ by cross-validated MANOVA. Neuroimage 89:345-357.

Axelrod V, Yovel G (2015) Successful decoding of famous faces in the fusiform face area. PLoS One 10:e0117126.

Axelrod V, Bar M, Rees G (2015) Exploring the unconscious using faces. Trends Cogn Sci 19:35-45.

Bar M, Tootell RB, Schacter DL, Greve DN, Fischl B, Mendola JD, Rosen BR, Dale AM (2001) Cortical mechanisms specific to explicit visual object recognition. Neuron 29:529-535.

Baria AT, Maniscalco B, He BJ (2017) Initial-state-dependent, robust, transient neural dynamics encode conscious visual perception. PLoS Comput Biol 13:e1005806.

Bastos AM, Usrey WM, Adams RA, Mangun GR, Fries P, Friston KJ (2012) Canonical microcircuits for predictive coding. Neuron 76:695-711.

Bastos AM, Vezoli J, Bosman CA, Schoffelen J-M, Oostenveld R, Dowdall JR, De Weerd P, Kennedy H, Fries P (2015) Visual areas exert feedforward and feedback influences through distinct frequency channels. Neuron 85:390-401. 
Boly M, Massimini M, Tsuchiya N, Postle BR, Koch C, Tononi G (2017) Are the Neural Correlates of Consciousness in the Front or in the Back of the Cerebral Cortex?. J NeuroSci 37:9603-9613.

Bosch SE, Jehee JFM, Fernandez G, Doeller CF (2014) Reinstatement of Associative Memories in Early Visual Cortex Is Signaled by the Hippocampus. J Neurosci 34:7493-7500.

Brown R, Lau H, LeDoux JE (2019) Understanding the Higher-Order Approach to Consciousness. Trends Cogn Sci 23:754-768.

Caballero-Gaudes C, Reynolds RC (2017) Methods for cleaning the BOLD fMRI signal. Neuroimage 154:128-149.

Christophel TB, Klink PC, Spitzer B, Roelfsema PR, Haynes JD (2017) The distributed nature of working memory. Trends Cogn Sci 21:111-124.

Cichy RM, Heinzle J, Haynes J-D (2012) Imagery and perception share cortical representations of content and location. Cereb Cortex 22:372-380.

Cichy RM, Pantazis D, Oliva A (2014) Resolving human object recognition in space and time. Nat Neurosci 17:455-462.

Davidson M, Persaud N, Maniscalco B, Mobbs D, Passingham R, Cowey A, Lau H (2010) Awareness-related activity in prefrontal and parietal cortices reflects more than superior performance capacity: a blindsight case study. J Vis 10:897.

Dehaene S (2014) Consciousness and the brain: deciphering how the brain codes our thoughts. New York: Viking.

Dehaene S, Changeux J-P (2011) Experimental and theoretical approaches to conscious processing. Neuron 70:200-227.

Dehaene S, Sergent C, Changeux J-P (2003) A neuronal network model linking subjective reports and objective physiological data during conscious perception. Proc Natl Acad Sci U S A 100:85208525.

Dehaene S, Changeux J-P, Naccache L, Sackur J, Sergent C (2006) Conscious, preconscious, and subliminal processing: a testable taxonomy. Trends Cogn Sci 10:204-211.

Del Cul A, Baillet S, Dehaene S (2007) Brain dynamics underlying the nonlinear threshold for access to consciousness. PLoS Biol 5: e260.

Dentico D, Cheung BL, Chang J-Y, Guokas J, Boly M, Tononi G, Van Veen B (2014) Reversal of cortical information flow during visual imagery as compared to visual perception. Neuroimage 100:237243.

Dijkstra N, Bosch SE, van Gerven MAJ (2017a) Vividness of visual imagery depends on the neural overlap with perception in visual areas. J Neurosci 37:1367-1373.

Dijkstra N, Zeidman P, Ondobaka S, van Gerven MAJ, Friston K (2017b) Distinct top-down and bottom-up brain connectivity during visual perception and imagery. Sci Rep 7:5677.

Dijkstra N, Mostert P, de Lange FP, Bosch S, van Gerven MAJ (2018) Differential temporal dynamics during visual imagery and perception. Elife 7:1-16.

Dijkstra N, Bosch SE, van Gerven MAJ (2019) Shared neural mechanisms of visual perception and imagery. Trends Cogn Sci 23:423434.

Dijkstra N, Ambrogioni A, Vidaurre D, van Gerven MAJ (2020) Neural dynamics of perceptual inference and its reversal during imager. eLife 9:e53588.

Eger E, Kell CA, Kleinschmidt A (2008) Graded size sensitivty of object exemplar-evoked activity patterns within human LOC subregions. J Neurophysiol 100:2038-2047.

Fahrenfort JJ, Scholte HS, Lamme VAF (2007) Masking disrupts reentrant processing in human visual cortex. J Cogn Neurosci 19:1488-1497.

Fahrenfort JJ, Snijders TM, Heinen K, van Gaal S, Scholte HS, Lamme VAF (2012) Neuronal integration in visual cortex elevates face category tuning to conscious face perception. Proc Natl Acad Sci U S A 109:21504-21509.

Fogelson SV, Kohler PJ, Miller KJ, Granger R, Tse PU (2014) Unconscious neural processing differs with method used to render stimuli invisible. Front Psychol 5:601.
Friston KJ, Buechel C, Fink GR, Morris J, Rolls E, Dolan RJ (1997) Psychophysiological and modulatory interactions in neuroimaging. Neurolmage 6:218-229.

Gardumi A, Ivanov D, Hausfeld L, Valente G, Formisano E, Uludağ K (2016) The effect of spatial resolution on decoding accuracy in fMRI multivariate pattern analysis. Neuroimage 132:32-42.

Harrison SA, Tong F (2009) Decoding reveals the contents of visual working memory in early visual areas. Nature 458:632-635.

He BJ (2018) Robust, transient neural dynamics during conscious perception. Trends Cogn Sci 22:563-565.

Hendriks MHA, Daniels N, Pegado F, Op de Beeck HP (2017) The effect of spatial smoothing on representational similarity in a simple motor paradigm. Front Neurol 8:222

Horikawa T, Kamitani Y (2017) Generic decoding of seen and imagined objects using hierarchical visual features. Nat Commun 8:15037.

Izatt G, Dubois J, Faivre N, Koch C (2014) A direct comparison of unconscious face processing under masking and interocular suppression. Front Psychol 5:659.

Jiang Y, He S (2006) Cortical responses to invisible faces: dissociating subsystems for facial-information processing. Curr Biol 16:2023-2029.

Johnson MR, Johnson MK (2014) Decoding individual natural scene representations during perception and imagery. Front Hum Neurosci 8:59.

King J-R, Pescetelli N, Dehaene S (2016) Brain mechanisms underlying the brief maintenance of seen and unseen sensory information. Neuron 92:1122-1134.

Kosslyn SM, Thompson WL (2003) When is early visual cortex activated during visual mental imagery? Psychol Bull 129:723-746.

Kovalenko LY, Chaumon M, Busch NA (2012) A pool of pairs of related objects (POPORO) for investigating visual semantic integration: behavioral and electrophysiological validation. Brain Topogr 25:272-284.

Lamme V (2015) The crack of dawn-perceptual functions and neural mechanisms that mark the transition from unconscious processing to conscious vision. In: Open mind, Vol 22 (Metzinger T, Windt JM, eds), pp 1-34. Frankfurt am Main, Germany: MIND Group.

Lamme VAF, Roelfsema PR (2000) The distinct modes of vision offered by feedforward and recurrent processing. Trends Neurosci 23:571-579.

Lamme VAF, Zipser K, Spekreijse H (2002) Masking interrupts figureground signals in V1. J Cogn Neurosci 14:1044-1053.

Lau HC, Passingham RE (2006) Relative blindsight in normal observers and the neural correlate of visual consciousness. Proc Natl Acad Sci U S A 103:18763-18768.

Lau H, Rosentha ID (2011) Empirical support for higher-order theories of conscious awareness. Trends Cogn Sci 15:365-373.

Lee S-H, Kravitz DJ, Baker Cl (2012) Disentangling visual imagery and perception of real-world objects. Neuroimage 59:4064-4073.

Lund TE, Nørgaard MD, Rostrup E, Rowe JB, Paulson OB (2005) Motion or activity: their role in intra- and inter-subject variation in fMRI. Neuroimage 26:960-964.

Macmillan NA, Creelman CD (1990) Detection theory: a user's guide. Mahwah, NJ: Lawrence Erlbaum.

Marks DF (1973) Visual imagery differences in the recall of pictures. Br J Psychol 64:17-24.

Mashour GA, Roelfsema P, Changeux JP, Dehaene S (2020) Conscious processing and the global neuronal workspace hypothesis. neuron. Neuron 105:776-798.

Mechelli A, Price CJ, Friston KJ, Ishai A (2004) Where bottom-up meets top-down: neuronal interactions during perception and imagery. Cereb Cortex 14:1256-1265.

Meijs EL, Mostert P, Slagter HA, de Lange FP, van Gaal S (2019) Exploring the role of expectations and stimulus relevance on stimulus-specific neural representations and conscious report. Neurosci Conscious 2019:niz011.

Misaki M, Luh WM, Bandettini PA (2013) The effect of spatial smoothing on $\mathrm{fMRI}$ decoding of columnar-level organization with linear support vector machine. J Neurosci Methods 212:355-361. 
Mostert P, Kok P, de Lange FP (2015) Dissociating sensory from decision processes in human perceptual decision making. Sci Rep 5:18253.

Moutoussis K, Zeki S (2002) The relationship between cortical activation and perception investigated with invisible stimuli. Proc Natl Acad Sci U S A 99:9527-9532.

Muckli L (2010) What are we missing here? Brain imaging evidence for higher cognitive functions in primary visual cortex V1. Int $\mathrm{J}$ Imaging Syst Technol 20:131-139.

O'Craven KM, Kanwisher N (2000) Mental imagery of faces and places activates corresponding stimulus-specific brain regions. $J$ Cogn Neurosci 12:1013-1023.

Pearson J (2019) The human imagination: the cognitive neuroscience of visual mental imagery. Nat Rev Neurosci 20:624-634.

Pearson J, Kosslyn SM (2015) The heterogeneity of mental representation: ending the imagery debate. Proc Natl Acad Sci U S A 112:10089-10092.

Pearson J, Naselaris T, Holmes EA, Kosslyn SM (2015) Mental imagery: functional mechanisms and clinical applications. Trends Cogn Sci 19:590-602.

Reddy L, Tsuchiya N, Serre T (2010) Reading the mind's eye: decoding category information during mental imagery. Neuroimage 50:818-825.

Rees G (2007) Neural correlates of the contents of visual awareness in humans. Philos Trans R Soc Lond B Biol Sci 362:877-886.

Reynolds JH, Heeger DJ (2009) The normalization model of attention. Neuron 61:168-185.

Roelfsema PR, Lamme VAF, Spekreijse H, Bosch H (2002) Figureground segregation in a recurrent network architecture. J Cogn Neurosci 14:525-537.

Schurger A, Pereira F, Treisman A, Cohen JD (2010) Reproducibility distinguishes conscious from nonconscious neural representations. Science 327:97-99.

Schurger A, Sarigiannidis I, Naccache L, Sitt JD, Dehaene S, Goldberg ME (2015) Cortical activity is more stable when sensory stimuli are consciously perceived. Proc Natl Acad Sci U S A 112: E2083-E2092.

Shanks D (2017) Regressive research: the pitfalls of post-hoc dataselection in the study of unconscious mental process. Psychon Bull Rev 24:752-775.

Stelzer J, Chen Y, Turner R (2013) Statistical inference and multiple testing correction in classification-based multi-voxel pattern analysis (MVPA): random permutations and cluster size control. Neuroimage 65:69-82.

Sterzer P, Rees G (2008) A neural basis for percept stabilization in binocular rivalry. J Cogn Neurosci 20:389-399.
Sterzer P, Haynes JD, Rees G (2008) Fine-scale activity patterns in high-level visual areas encode the category of invisible objects. $J$ Vis 8(15):10, 1-12.

Stokes M, Thompson R, Cusack R, Duncan J (2009) Top-down activation of shape-specific population codes in visual cortex during mental imagery. J Neurosci 29:1565-1572.

Thirion B, Duchesnay E, Hubbard E, Dubois J, Poline J-B, Lebihan D, Dehaene S (2006) Inverse retinotopy: inferring the visual content of images from brain activation patterns. Neuroimage 33:1104-1116.

Tong F, Meng M, Blake R (2006) Neural bases of binocular rivalry. Trends Cogn Sci 10:502-511.

Tononi G (2008) Consciousness as integrated information: a provisional manifesto. Biol Bull 215:216-242.

Tononi G, Koch C (2008) The neural correlates of consciousness: an update. Ann N Y Acad Sci 1124:239-261.

Tzourio-Mazoyer N, Landeau B, Papathanassiou D, Crivello F, Etard O, Delcroix N, Mazoyer B, Joliot N (2002) Automated anatomical labeling of activations in SPM using a macroscopic anatomical parcellation of the MNI MRI single-subject brain. Neuroimage 15:273-289.

van Gaal S, Lamme VAF (2012) Unconscious high-level information processing. Neuroscientist 18:287-301.

van Gaal S, Ridderinkhof KR, Fahrenfort JJ, Scholte HS, Lamme VAF (2008) Frontal cortex mediates unconsciously triggered inhibitory control. J Neurosci 28:8053-8062.

van Gaal S, Lamme VAF, Fahrenfort JJ, Ridderinkhof KR (2011) Dissociable brain mechanisms underlying the conscious and unconscious control of behavior. J Cogn Neurosci 23:91-105.

van den Hurck J, Op de Beeck H (2019) Generalization assymetry in multivariate cross-classification: when representation $A$ generalizes better to representation $B$ than $B$ to $A$. BioRXiv. Advance online publication. Retrieved September 30, 2021. doi:.

Weaver MD, Fahrenfort JJ, Belopolsky A, Van Gaal S (2019) Independent neural activity patterns for sensory-and confidencebased information maintenance during category-selective visual processing. Eneuro 6:ENEURO.0268-18.2018.

Wyart V, Nobre AC, Summerfield C (2012) Dissociable prior influences of signal probability and relevance on visual contrast sensitivity. Proceedings of the National Academy of Sciences of the United States of America 109:3593-3598.

Xie S, Kaiser D, Cichy RM (2020) Visual imagery and perception share neural representations in the alpha frequency band. Curr Biol 30:2621-2627.

Zeidman P, Jafarian A, Seghier ML, Litvak V, Cagnan H, Price CJ, Friston KJ (2019) A guide to group effective connectivity analysis, part 2: Second level analysis with PEB. Neurolmage 200:174-190. 